\title{
Marginalization and Exclusion: Unraveling Systemic Bias in Classification
}

Mai, Jens-Erik

Published in:

Knowledge Organization

Publication date:

2016

Citation for published version (APA):

Mai, J-E. (2016). Marginalization and Exclusion: Unraveling Systemic Bias in Classification. Knowledge Organization, 43(5), 324-330. 


\title{
Marginalization and Exclusion: Unraveling Systemic Bias in Classification
}

\author{
Jens-Erik Mai \\ University of Copenhagen, Royal School of Library and Information Science, 6 Birketinget, \\ DK-2300 Copenhagen S, Denmark, <jenserikmai.info>, <jemai@hum.ku.dk>
}

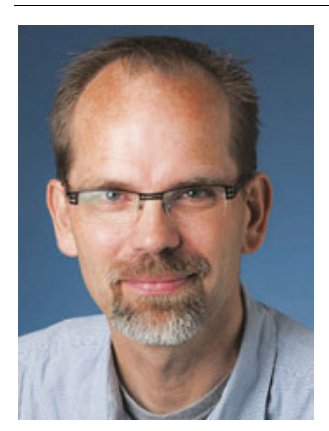

Jens-Erik Mai is Professor of information studies at the University of Copenhagen. His work concerns basic questions about the nature of information phenomena in contemporary society-he is concerned with the state of privacy and surveillance given new digital media, with classification given the pluralistic nature of meaning and society, and with information given its pragmatic nature. He teaches courses on knowledge organization, information ethics, privacy and surveillance, and knowledge media. He earned his PhD from the University of Texas at Austin as a Fulbright Scholar and his masters and bachelors degrees from the Royal School of Library and Information Science, Denmark.

Mai, Jens-Erik. 2016. "Marginalization and Exclusion: Unraveling Systemic Bias in Classification.” Knowledge Organization 43: 324-330. 25 references.

Abstract: This paper explores the knowledge organization landscape in whi butions to the field are situated. The paper first explores some of the foundational conceptual notions within knowledge organization that today are well-accepted. The paper then reviews Hope Olson's contributions to that landscape and places her work in the context of Borges' essay about John Wilkins' project to develop a universal language that would organize and contain all human thought. The paper shows how Hope Olson's work on marginalization and exclusion of specific topics and groups of people in large library classification has unraveled the systemic bias found in all classifications. The paper calls for stronger engagement between scholarship and practice to address marginalization and exclusion in further work on classification systems.

Received: 17 November 2015; Revised 28 February 2016; Accepted 14 March 2016

Keywords: classification, knowledge organization systems, libraries, Hope Olson, Borges

\subsection{Introduction}

In his short essay, The Analytic Language of John Wilkins, Borges $(1942 / 1973,102)$ tells the story of John Wilkins, who in 1664 undertook the task to develop a "universal language that would organize and contain all human thought." Wilkins divided the universe into "forty categories," which again can be further divided into first "differences" and then into "species." Each word in this language would be meaningful and hold the definition of the concept to which it refers and as such be a sort of an encyclopedia of all human knowledge. After pondering over Wilkins' universal language, the fictitious but famous Celestial Emporium of Benevolent Knowledge, and the work of The Bibliographic Institute of Brussels, Borges suggests (104) that, "obviously there is no classification of the universe that is not arbitrary and conjectural" because, as Borges says, "The reason is very simple: we do not know what the universe is." This fact, that we cannot know the universe, however, "cannot dissuade us from outlining human schemes, even though we are aware that they are provisional." While there are needs to devise classification systems for a variety of reasons, including to facilitate search, browsing, and retrieval in libraries, Borges suggests that, "we must conjecture its purpose; we must conjecture the words, the definitions, the etymologies, the synonymies of God's secret dictionary."

What made Borges' article famous and widely cited was the Celestial Emporium of Benevolent Knowledge that apparently stems from a "certain Chinese encyclopedia" (Borges [1942] 1973, 103), which divided animals into 14 categories:

(a) Those that belong to the Emperor, (b) embalmed ones, (c) those that are trained, (d) suckling pigs, (e) mermaids, (f) fabulous ones, (g) stray dogs, (h) those that are included in the this classification, (i) those that tremble as if they were mad, (j) innumerable ones, $(\mathrm{k})$ those drawn with a very fine camel's hair brush, (l) others, (m) those that have just broken a flower vase, (n) those that resemble flies from a distance. 
A number of authors have used this set of categories and Borges' essay as a springboard to discuss classification, the construction of categories, and issues of representation. Foucault ([1966] 1970) laughed for "a long time" when he first read Borges' essay, but, as he reports (xvii), it was "not without a certain uneasiness that I found hard to shake off," because this unfamiliar classification of animals allows us to reflect upon our own classifications and systems of order. Borges' classification of animals allows us to wonder about such a different system of thought and our "stark impossibility of thinking that' (xv) and thereby exposes the limitations of our own systems of order and thinking. Layoff (1987) picks up the same reading of exposing our own cultural biases when faced with odd classifications; Borges's classification of animals (92), "comes close to the impression a Western reader gets when reading descriptions of nonwestern languages and cultures." It is a good example of classifications that "boggle the Western mind" and leave anthropologists and linguists with no explanations.

Bowker and Star $(1999,131)$ use Borges' classification of animals to make the point that "classifications that appear natural, eloquent, and homogenous within a given human context appear forced and heterogeneous outside that context;" only outside their contexts will the forced nature of classifications be revealed. The arbitrary and conjectural nature of all classifications is revealed when they are taken outside their context and as such the contextuality of classifications is emphasized. Weinberger (2007) uses Borges' division of animals to make a few practical observations about the production of classifications and lists in general. First, "Borges is able to ignore the "A list is a list of something' rule" (66), in other words the point is that any classification, any list, is produced for a particular purpose and reason to serve a specific community and context. Secondly, Weinberger finds that Borges violates the principle of having all categories at the same hierarchical level at the same level of abstraction; "Borges includes 'stray dogs,' 'having just broken the water pitcher,'1 and 'innumerable' as if they were all at the same level of generality" (66). Duszat $(2012)^{2}$ provides an excellent analysis of Borges' essay in which he discusses the elements of the essay, traces the origins of the arguments, and lays the foundation for a thorough understanding of Borges' essay and especially Foucault's interpretation of it.

What is common for these contemporary discussions of classification is that they use Borges' text as a way to analyze and discuss classification practice and theory; they all view classification as both concrete manifestations and conceptual constructions at the same time. Practical implementations cannot be separated from the thoughts and decisions that have gone into devising the system. As such the authors highlight the limitations of our own systems of thought, the cultural biases of classifications, the forced na- ture of classifications, the observation that classifications are always created with a purpose in mind, and the challenges with the expectation that we have come to expect symmetries in the levels of abstraction across categories at the same level.

That Borges' points are taken to be obvious and almost without exception would be accepted across the vast majority of scholars in the social sciences and humanities todayand the points are now gaining traction within the natural sciences (cf. e.g., Bryant 2000, Dupré 1993, and Ereshefsky 2001). However, one must wonder why it is necessary to discuss the validity and soundness of these points within the context of library classification. In his work on the history and practice of library classification, Miksa $(1998,81)$ suggests that one common goal and hope for much work in library classification has been that, "somewhere, somehow, we can, or should try to, produce the one best classification system that will serve all purposes" with (Miksa 2009, 39) "the ideal of achieving international bibliographic control." Patrick Wilson $(1983,65)$ had earlier questioned this goal of the one best system and observed that:

If we admit that the number of different perspectives from which the world can be viewed and described is endless, we shall expect that the library to contain competing, conflicting accounts of the world that cannot be incorporated into a single consistent story of the way things are.

There seems to be a tension between conceptual work on library classification on the one hand and on the other hand the application and employment of classification schemes in libraries and especially among the developers of large universal library classification systems. That tension seems to hinder a meaningful and fruitful crosscollaboration and exchange of ideas, knowledge, and experiences between research and practice. Following Miksa's (1998) reasoning, it could be suggested that this tension has been present since the early days of modern library classification (starting in the 1880s, and developing in the early twentieth century). Miksa finds that the connection between, on one hand, philosophers and others concerned with the structure, organization, and theories of knowledge, and, on the other hand, the practice of devising and maintaining library classifications, is "actually really weak" (36). Miksa argues that while the philosophical pursuit to organize knowledge was a means to understand the world and the knowledge of the world, "the goal of librarians was no less important than the goal of the classificationists of the knowledge of the sciences, but it had a different purpose" and this different purpose helped librarians focus on the practical problems at hand, and not get lost in philosophical theorizing (40): 
Library classificationists had a much more practical, though no less exalted, task in view. That more practical task was to make the knowledge found in books accessible to people - in fact, not simply the knowledge found in any books but the knowledge found in the best books by the best authors.

In fact this single-minded focus on the practicalities of the task at hand led these early library classificationists to assume that the (46) "task had everything to do with practicality and very little to do with philosophical speculations about knowledge."

\subsection{Speculating about knowledge}

Wilson $(1968,21)$ also suggested that knowledge organization is driven by the practicality of providing users with "the best textual means to his end." In Wilson's vocabulary, this ability requires that librarians and knowledge organization systems have a special power, namely the (22) "power to produce the best textual means to one's ends." To help explore this ability to determine and retrieve the best textual means, Wilson distinguished between two kinds of power; a descriptive power and an exploitative power. Descriptive power is the ability to retrieve all the documents that fit a certain description, "all those written by Hobbes, all those doctrine of eternal recurrence, all those containing the word 'fatuity"' and as such the descriptive power is "evaluatively neutral;" it is merely (25) "the ability to line up a population of writings in any arbitrary order, to make the population march to one's command."

Exploitative power, on the other hand, is the ability (25) "to merely say what [the writings are] for, and [the user] is then provided with what will suit that purpose best, whatever it is." This sort of power requires capabilities beyond neutral, objective and detached descriptions of documents; it requires that librarians and knowledge organization systems have (22) a "power [that] essentially involve[d] the appraisal of writings ... the estimation of the merits and defects of writings in terms of credibility, of intelligibility, of accuracy, of adaptability or utility, of scholarship, and so on." Of these two powers, it is obvious that the exploitative is more attractive; who would want descriptive power if one could have exploitative power?

The point is, of course, that the two powers are not independent; one cannot have one without the other. It is impossible to retrieve all items that fit a description without some evaluation of the relation between what is needed for the user's particular situation, and it is not possible to have exploitative power without some description of the items regardless of particular interests and situations. All knowledge organization systems have elements of both powers ingrained into their design, implementa- tion, and use. In other words, the very fundation of knowledge organization systems demands that there is a balancing between designing and using the system for and within a particular context, while at the same time striving towards making the system universally applicable.

While Wilson ignited a discussion in the knowledge organization community about the purpose, aim, and goals of knowledge organization systems and practice, that discussion and that search for conceptual and theoretical foundation is still ongoing. Furner (2010) recently observed that knowledge organization has now reached a stage with two distinct positions, or rather two ends of a spectrum. At one end are "nominalists" (186) who regard subjects, classifications, and representation to be contextual and situationally bound; that there is no one true correct way to construct a classification and no one true way to represent a document. The nominalists often directly or indirectly aim for Wilson's exploitative power. At the other end of the spectrum are the "realists" who hold that the subject matter of documents exists independently of humans and of culture, space, and time and that a classification ought to apply to any and all contexts and situations. The realists aim for Wilson's descriptive power. The discussions among these positions do not seem to come to a conclusion and the discussions do not seem to have brought the two ends of the spectrum closer to a conclusion, as Furner notes (2010, 186-7):

The fact that, at this point in the history of theory in information studies, the nature of aboutness continues to be the subject of such debate in the field is a result not primarily of the inability of proponents of views at the two poles to persuade their opponents of the merits of those views, but rather of the largely unacknowledged influence of the realist view on the activity of designers and users of knowledge organization systems. It is difficult to find wellreasoned defenses of the realist view in the literature, yet most of us who are actively engaged in the tasks of designing bibliographic classification schemes, indexing documents in accordance with such schemes, and using those schemes as tools for finding documents of the kinds that we want, continue to act as if we accept the realist view as the correct one.

Some of this acting as if we accept the realist position has to do with the language employed in knowledge organization. We talk about the "exhaustivity" in subject analysis, the "specificity" of an index term, the "facets of a subject," the "subject matter of a document," etc. In all these instances we employ, a "realist" vocabulary to discuss the activities and entities in knowledge organization; we talk about the representation and organization of subjects as if 
the subjects were tangible entities that can be moved around and placed in classes.

The thinking and theoretical foundation for classification that Borges' essay explores, and with which the authors who have used Borges' essay as a springboard for their investigations into classification, is in line with the majority of scholarship across the social sciences and humanities as it has played out at least since the "linguistic turn" in the middle of the twentieth century (Rorty 1967). It is therefore curious that the terminology, conceptual foundation, and thinking about knowledge organization seems to still rest in what Furner calls a realist paradigm. This could suggest that the literature and practice within knowledge organization simply have yet to reorient the theoretical foundation from past times to more contemporary theories and thinking. However, as Miksa has demonstrated, that challenge could be even deeper; the reason for the practice's lack of interest in contemporary theories might be due to the practice's lack of theoretical foundation in general. It is difficult to have a dialog between research and practice when they speak two different languages; where research and scholarship is concerned with the discovery of new insights and knowledge about knowledge organization, practice seems concerned with the practical task at hand of designing, implementing, and maintaining already existing knowledge organization systems.

It is in this landscape of somewhat naive realism with a focus on practical solutions without regard to their theoretical foundation or the lack thereof on one hand, and on the other hand an increasing theoretical awareness of the epistemological and ethical challenges that classification face after the linguistic turn, that Hope Olson enters knowledge organization research after having been a practitioner. She entered the field speculating about knowledge with a baggage of practice and a deep desire for cultural change.

\subsection{Cultural change}

Hope Olson enters the discussion of library classification with a basic concern about the ethical and professional core belief that libraries, librarians, and knowledge organization systems ought to become "“neutral' intermediaries" (Olson 2001b, 640). She argues that this core belief has led to a "pervasive belief among information scientists that in order to create an overriding unity in language the diversity and subjectivity of language need to be standardized." She continues her critiques of this core belief with a statement that makes these information scientists sound as if they have taken inspiration from John Wilkins' project to develop a universal language of all human thought: "Librarians call such a constructed universal language a controlled vo- cabulary." The main challenge, according to Hope Olson, is that this commitment to neutrality cloak the biases of classifications and hinder conversations about the cultural effects of classifications that marginalize and exclude views outside of the political, social, and ethical mainstream. This core belief among information scientists and librarians that they should remain neutral and the belief that knowledge organization systems ought to be neutral and universal has brought about the understanding that knowledge organization systems:

Appear unbiased and universally applicable-but they actually hide their exclusions under the guise of neutrality ... [which] disproportionally affects access to information outside of the cultural mainstream and about groups marginalized in our society.

While there have been critiques in the literature of library classification systems' treatment of “women, Puerto Ricans, Chinese and Japanese Americans, Mexican Americans, Jews, Native Americans, the developing world (including Africa, the Middle East, and Melanesia), gays, teenagers, senior citizens, people with disabilities, and alternative lifestyles" (Olson 1998, 34) the problem remains. Hope Olson suggests that it may be difficult to identify such marginalization and exclusion of groups of people because library classifications often reflect a mainstream cultural position: "The categories of classification-because they typically reflect a cultural mainstream-appear neutral, objective, and transparent. This makes marginalizations and exclusions difficult to identify."

Regardless that it may be difficult to identity and expose these biases, Hope Olson remains steadfast that the work is necessary and important. Again, her own position and approach is one that starts from within the library and knowledge organization practitioner community; she identifies with practice and argues from within practice. Yet, she is adamant that the challenges of systemic biases in library classification can be changed, and need to change (Olson 2001b, 639):

Certainly libraries, like other institutions, reflect the marginalization and exclusions of the society they serve. After twenty-five years of studying, doing, managing, and teaching cataloging and classification, I find the problem both acute and systemic but also amendable to change.

Instead of the traditional library values of neutrality and universality, Hope Olson suggests a foundation that is based on plurality and diversity (Olson 2001a, 21): "I would like to build on the idea of diversity rather than universality as a way to accommodate our diverse users and collections 
and thus, decreasing levels of bias." Hope Olson takes the challenge one step further. She argues that not only do library classifications need to change and become inclusive of the world's diversity but library classifications can lead to a cultural change in mainstream culture. Once library classifications have changed and become inclusive and accommodating to diversity, library classification might serve as agents to affect society and culture, "a mainstream tool such as a standard library controlled vocabulary is potentially an agent of cultural change" (Olson 2000, 54).

Hope Olson uses her deep knowledge of both theory and practice throughout her scholarship. Take for instance her close reading of Dewey Decimal Classification (DDC) and Library of Congress Subject Headings (LCSH) classifications for eleven specific books that $(2002,184)$ :

Combine a feminist perspective with attention to particular groups of women identifying with one or more of the following: women of colour, African American women, Chicanas, lesbians, Asian American women, working class women, Jewish women, North American Aboriginal women.

In her reading and discussion of the specific entries of the books, Hope Olson demonstrates how decisions have been made that marginalize and exclude the books' subject matter from classifications' mainstream culture. She does this by discussing each heading and class number assigned to the books and exploring the systems to understand the specific meaning of those headings and class numbers within the systems. She further suggests other supplementary headings and class numbers that might have been assigned to better represent the books' subject matter or perspective and capture the books' diversity. Her conclusion is rather harsh. She suggests that the poor quality of the $D D C$ and LCSH entries is a reflection of society's lack of interest in these books and their subject matter; the books' subject matter "is separated from the mainstream and then pushed aside where it will not disturb library users looking for books on real topics" (223). Hope Olson reaches this conclusion due to two interrelated challenges with both $D D C$ and LCSH: both systems have a systemic lack of vocabulary to capture the subject matter of such books, and the systems lack the ability to express the diversity of such books' subject matter. DDC and LCSH's "consistent marginalization and exclusion in the representation of Other women" (222) stems from the tradition of striving for neutrality and universality in the practical application of library classification that, as Miksa $(1998,81)$ noted, seeks to "produce the one best classification system that will serve all purposes."

In another close reading, Hope Olson explores the basic "presumption that a system can be universal in cover- age" (Olson 2000, 44). This work is based on the principled observation (Olson 2007, 509) that "the organization of information ... is primarily based on traditional or Aristotelian logic.” As Hope Olson demonstrates, a large amount of classification work in and outside library classification builds on basic principles from classic logic, and this tradition has helped formalize structures and organization of information and scientific objects and discoveries and as such has much to offer. The challenge, therefore, is not classic logic in itself but the application of logic in classification. In other words, it is important to "reject any notion that traditional/Aristotelian logic is the logical structure" and instead view "it as a logical structure" (522). With this conceptual view of logic, Hope Olson explores a number of sections and classificatory structures in DDC and LCSH. In each case, Hope Olson demonstrates how the classification systems represent a particular view of the world, and how they marginalize and exclude other views of the world. Hope Olson's analyses show that the construction of structures and classes in classification systems are not bound by strict Aristotelian logic but are constructed views and interpretations of the world; not in the sense of being universal and inclusive but as particular views of the world. Hope Olson shows that other views and other interpretations are possible and as such there can be no true universal classification system. Hope Olson's analysis and discussion of DDC and LCSH is in line with Borges's observation that such systems are "provisional" (Borges [1942] 1973 , 104) and that "we must conjecture its purpose; we must conjecture the words, the definitions, the etymologies, the synonymies of God's secret dictionary."

While Hope Olson's analysis and interaction with the $D D C$ and $L C S H$ reveal its biases and the marginalization and exclusions these biases entail, the main frustration might not be with the biases themselves but with the reaction from these library classification systems. Although Hope Olson notes that $(2002,22)$, "the editors of DDC and of other classifications regularly respond to concerns about bias at the same time that they endeavor to make their changes manageable for existing collections," the main challenge is that the editors have not seriously entered the theoretical conversation about cultural biases in their systems and their marginalization and exclusions of certain groups of people and viewpoints. As Feinberg (2011) has pointed out, the problem is not the systems' bias-the challenge is that the systems are not open about the positions they take, their view of the world, and their interpretive stance (122):

I would say that the DDC, or any classification, is not problematic if it expresses a point of view on the subject matter that it organizes. Indeed, there is 
no way it can avoid doing so. The problem is that when the DDC, or any classification, is not forthright about its position, when it doesn't acknowledge its own rhetorical effects, when it hides behind an assumed tool-nature to avoid coming to terms with its document-nature.

In fact, this is not just a matter of articulating the point of view that the library classification systems take about the world, it is an ethical matter. A matter that is too serious to skate over and not take seriously. Bowker and Star (1999, 5-6) take Hope Olson's argument one step further, and suggest that all classification "valorizes some point of view and silences another. This is not inherently a bad thingindeed it inescapable. But it is an ethical choice, and as such it is dangerous-not bad, but dangerous."

That dangerous ground is made up of ethical concerns that force one to take a stand, and the editors of large universal library classification systems appear curiously unprepared to enter the discourse about the epistemological and ethical foundation of their systems. Furner (2007) — very much in the spirit of Hope Olson's close readings of DCC and LCSH - analyzed DDCs Table 5 "Racial, Ethnic, National Groups/Ethnic and National Groups" and the changes that were made in 2003 to the table and to classification practice with regards to works on race-he charged (165) that the editors of the DDC: "conferred a weighty responsibility: the moral obligation to do what can be done to eradicate the racism by which, simply as contemporary Western institutions, classification schemes are unarguably infected." Characteristic for the misconstrued dialog between scholars and practice, Furner's analysis and call for action was met with a technical paper in which the editors proposed a few technical solutions to the ethical dilemma in which the DDC was caught (Beall 2009). As Miksa $(1998,46)$ noted, library classificationists in the early twentieth century perceived that their "task had everything to do with practicality and very little to do with philosophical speculations about knowledge." It appears that situations remain unchanged a century later; library practitioners appear to be concerned with practicalities rather than scholarship about knowledge and knowledge organization.

\subsection{Conclusions}

Hope Olson has made three significant contributions to knowledge organization. First and foremost, she has unraveled the systemic bias in classification by shining a light on the widespread marginalization and exclusion of topics and groups of people outside the cultural mainstream. The many examples that Hope Olson has analyzed would surely have made Foucault "laugh" the same way he laughed when he read Borges' essay, and Lakoff would surely have been "boggled" just as he finds Westerns boggled by Borges' classification of animals. Hope Olson has demonstrated the "arbitrary and conjectural" (Borges [1942] 1973, 104) nature of large library classifications, and the unfortunate consequences of the deep but "largely unacknowledged influence of the realist view on the activity of designers and users of knowledge organization systems“ (Furner 2010, 87).

Secondly, through her scholarship, Hope Olson has developed a methodological approach to close readings and analysis of classificatory structures and classification of books, which allows her to unravel the decisions and systemic biases in library classification systems. The methodological approach is open for others to continue her work. And thirdly, Hope Olson has inspired a new generation of knowledge organization scholars, brought up with the notion that all classifications are biased. What is unique about the new generation of knowledge organization scholars is that they have accepted that knowledge organization ought to aim for plurality and diversity. Hope Olson's vast contributions to knowledge organization demonstrate the systemic bias in classifications, and have laid the foundation to explore the social influences on classification.

While today it is widely accepted that classifications are influenced by their societal contexts, scholarship on this topic was formed in the early 1970s. In Hope Olson's (2010) entry in the Encyclopedia of Library and Information Sciences, she gives a short review of the relatively few works on the social influences on classification. The review reveals that this work almost universally comes to the same conclusion, namely that there is a bias in library classification systems that marginalizes and excludes specific topics and groups of people. However, while it is now accepted that there is a social influence on classification, Hope Olson had originally suggested that classifications could potentially be "an agent of cultural change" (Olson 2000, 54). This, however, remains to be explored (Olson 2010, 4811): "That classification is an artifact crafted in a social context is evident. More difficult to assess is whether or not the reverse is the case. Does classification, in turn, influence a society?"

Contemporary scholarship on classification has sometimes used Borges' essay about John Wilkins' universal language to organize and contain all human thought as a springboard to discuss and analyze classification practice. The similarities between John Wilkins's project and the universal library classification movement are striking; they both aim to describe the world independent of time and space, they both aim to create one system applicable to all contexts and cultures, and they both strive for neutrality and objectivity. Contemporary scholarship on classification almost universally takes a different stand on these issues; it 
is now accepted that classifications are culturally biased, that classifications appear forced and heterogeneous outside their context, and that classifications are always created for a specific purpose. Hope Olson has played a central and significant role in the development of the theoretical foundation for analysis of classifications - and her work might inspire practitioners to reconsider their positions and enter the dialog about the design and development of classifications in the 21 st century.

\section{Notes}

1. The various English translations of Borges' essay vary in the translation of some of the entries in the classification of animals; in some translations the broken item is a "flower vase," other times it is a "water pitcher."

2. Thanks to one of the anonymous reviewers for the reference to Duszat's paper.

\section{References}

Beall, Julianne. 2009. "Racially Mixed People, DDC Table 5 Ethnic and National Groups, and MARC 21 Bibliographic Format Field 083." Cataloging and Classification Quarterly 47, no. 7:657-70.

Borges, Jorge Luis. (1942) 1973. "The Analytical Language of John Wilkins.” In Other Inquisitions 1937-1952, trans. Ruth L. C. Simms. London: Souvenir Press, 101-5.

Bowker, Geoffrey, and Susan Leigh Star. 2000. Sorting Things Out: Classification and Its Consequences. Cambridge, MA: MIT Press.

Boyne, Roy. 2006. "Classification." Theory, Culture and Society 23, nos. 2-3:21-30.

Bryant, Rebecca. 2000. Discovery and Decision: Exploring the Metaphysics and Epistemology of Scientific Classification. Cranbury, NJ: Associated University Presses.

Dupré, John. 1993. The Disorder of Things: Metaphysical Foundations of the Disunity of Science. Cambridge, MA: Harvard University Press.

Duszat, Michael. 2012. "Foucault's Laughter: Enumeration, Rewriting, and the Construction of the Essayist in Borges's 'The analytical language of John Wilkins'." Orbis Litterarum 67:193-218.

Ereshefsky, Marc. 2001. The Poverty of the Linnaean Hierarchy: A Philosophical Study of Biological Taxonomy. New York: Cambridge University Press.

Feinberg, Melanie. 2011. "Organization as Expression: Classification as Digital Media." In Digital Media: Technological and Social Challenges of the Interactive World, ed.
Megan A. Winget and William Aspray. Lanham: Scarecrow Press, 115-33.

Foucault, Michel. (1966) 1970. The Order of Things: An Archaeology of the Human Sciences. New York, NY: Vintage Books.

Furner, Jonathan. 2010. "Philosophy and Information Studies." Annual Review of Information Science and Technology 44:161-200. doi:10.1002/aris.2010.1440440111

Furner, Jonathan. 2007. "Dewey Deracialized: A Critical Race-theoretic Perspective." Knowledge Organization 24:144-68.

Lakoff, George. 1987. Women, Fire and Dangerous Things: What Categories Reveal about the Mind. Chicago: University of Chicago Press.

Miksa, Francis. 2009. "A Review Article: Chan, Taylor, and the Future of Cataloging Texts." Library Quarterly 79:131-43.

Miksa, Francis. 1998. The DDC, the Universe of Knowledge, and the Post-Modern Library. Albany, NY: Forest Press.

Olson, Hope A. 1998. "Mapping Beyond Dewey's Boundaries: Constructing Classificatory Space for Marginalized Knowledge Domains." Library Trends 47, no. 2:233-54.

Olson Hope A. 2000. "Difference, Culture and Change: The Untapped Potential of LCSH." Cataloging and Classification Quarterly 29, nos. 1-2:53-71.

Olson, Hope A. 2001a. "Sameness and Difference: A Cultural Foundation of Classification." Library Resources \& Technical Services 45:115-22.

Olson, Hope A. 2001b. "The Power to Name: Representation in Library Catalogs." Signs: Journal of Women in Culture and Society 26:639-68.

Olson, Hope A. 2002. The Power to Name: Locating the Limits of Subject Representation in Libraries. Dordrecht, The Netherlands: Kluwer Academic Publishers.

Olson, Hope A. 2007. "How We Construct Subjects: A Feminist Analysis.” Library Trends 56:509-41.

Olson, Hope A. 2010. "Social Influences on Classification." In Encyclopedia of Library and Information Sciences, 3rd ed., ed. Marcia J. Bates and Mary Niles Maack. New York: Taylor and Francis, 4806-13.

Rorty, Richard, ed. 1967. The Linguistic Turn: Recent Essays in Philosophical Method. Chicago: The University of Chicago Press.

Wilson, Patrick. 1983. Second-Hand Knowledge: An Inquiry into Cognitive Authority. Westport, Conn.: Greenwood.

Wilson, Patrick. 1968. Two Kinds of Power: An Essay on Bibliographical Control. Berkeley, CA: University of California Press. 